PAPER

\title{
Monitoring method of scattering at specimen in high-frequency measurement of normal-incidence sound-absorption-coefficient utilizing impedance tube
}

\author{
Akira Sanada $^{1, *}$ and Hiroshi Nakagawa ${ }^{2}$ \\ ${ }^{1}$ Industrial Technology Center of Okayama Prefecture, \\ 5301 Haga, Kita-ku, Okayama, 701-1296 Japan \\ ${ }^{2}$ Nihon Onkyo Engineering Co., Ltd., \\ 1-21-10 Midori, Sumida-ku, Tokyo, 130-0021 Japan
}

(Received 1 October 2018, Accepted for publication 15 February 2019)

\begin{abstract}
This study deals with development of a high-frequency measurement method of normalincidence sound absorption coefficient in a cylindrical impedance tube. The authors have previously proposed the method using multiple microphones which enables the normal-incidence absorption coefficient measurement in the frequency range beyond the cut-on frequencies of the higher order acoustic modes. It is confirmed that the proposed method is valid for commonly used absorbing materials at which the scattering does not occur. However, it is difficult to judge whether the scattering occurs or not, only from measured absorption coefficient. Hence, in this study, a method that evaluates the effect of the scattering is proposed to confirm the reliability of the measured results. As an indicator of the scattering effects, a normal-incidence $(1,0)$-mode-reflection coefficient and a total power reflection coefficient in a tube are defined. They are derived from the difference between the signals of the two microphones placed on opposite sides of the tube. Numerical simulations were conducted to prove the theory. Finally, the experimental results are shown, confirming the validity and the feasibility of the proposed method.
\end{abstract}

Keywords: Normal-incidence sound absorption coefficient, Acoustic impedance tube, Transferfunction method, Higher-order acoustic mode, Scattering

PACS number: 43.55.Ev [doi:10.1250/ast.40.250]

\section{INTRODUCTION}

The two-microphone transfer-function method using an acoustic impedance tube [1] is commonly used to measure the normal-incidence sound absorption coefficient of materials. The frequency measurable by the method is limited by the diameter of the tube because the measurement must satisfy the condition that only a normalpropagating wave exists in the tube. Above the cut-on frequencies of the higher order acoustic modes, not only the normal-propagating wave but also obliquely-propagating waves can exist. Hence, in that frequency range, the sound field in the tube becomes too complicated to measure the normal-incidence absorption coefficient with the twomicrophone transfer-function method. On the other hand, in order to decompose acoustic modes in a tube, the methods to measure a reflection matrix, which expresses

*e-mail: akira_sanada@pref.okayama.lg.jp the relation between the incident acoustic modes and the reflected acoustic modes in the tube, were proposed by some researchers. Åbom, M. proposed the method, by which sound waves in a cylindrical tube are identified using multiple microphones [2]. Schultz, T. et al. developed a method of measuring the reflection coefficient matrix for multiple acoustic modes in a rectangular duct using eight microphones [3]. They calculated all the elements of the reflection coefficient among the acoustic modes, including the reflection coefficient of the reflected $(0,0)$ mode to the incident $(0,0)$ mode, which corresponds to the normal-incidence reflection coefficient. However, these methods are complicated, even when measuring just the normal-incidence absorption coefficient, because they require multiple measurements for multiple sound sources and inverse matrix calculation. Therefore, the author proposed a simple practical method to measure normalincidence sound absorption coefficient at frequencies beyond the cut-on frequency of higher-order modes using 
four or eight microphones under an assumption that a large amount of scattering on the specimen surface does not exist [4,5]. In order to extract the normal propagating wave factor through a cross section of a cylindrical tube, four microphones are located with one in each quarter of the circumference and their signals are summed. The normalincidence absorption coefficient is calculated from the frequency response function between the normal-propagating factors of two cross sections, which are placed at a prescribed distance. However, when scattering at the specimen is large, the normal-incidence absorption coefficient cannot be measured accurately because the normalincidence wave is converted to obliquely reflected waves and the amount of normal-reflected waves decreases. Therefore, the proposed method requires the specimen at which the scattering does not occur, whereas, whether the scattering occurs or not might not be able to be judged only from the measured results.

Hence, in this study, a monitoring method of the scattering is proposed to confirm the reliability of measured results. As an indicator of the scattering effect, a normalincidence $(1,0)$-mode-reflection coefficient and a total power reflection coefficient in a tube are defined, considering the sound pressure difference between the signals of the two microphones placed on opposite sides of the tube. By monitoring these reflection coefficients, measurement can be performed as confirming the reliability of the measured results. First, the normal-incidence $(1,0)$-modereflection coefficient and a total power reflection coefficient in a tube are theoretically derived. Then, simulation and experimental results are shown, confirming the validity of the proposed method.

\section{THEORY}

\subsection{Extracting $(1,0)$ Mode Signal}

In this study, a cylindrical tube with a radius $R$ (Fig. 1) is considered. The sound pressure in the tube is expressed as

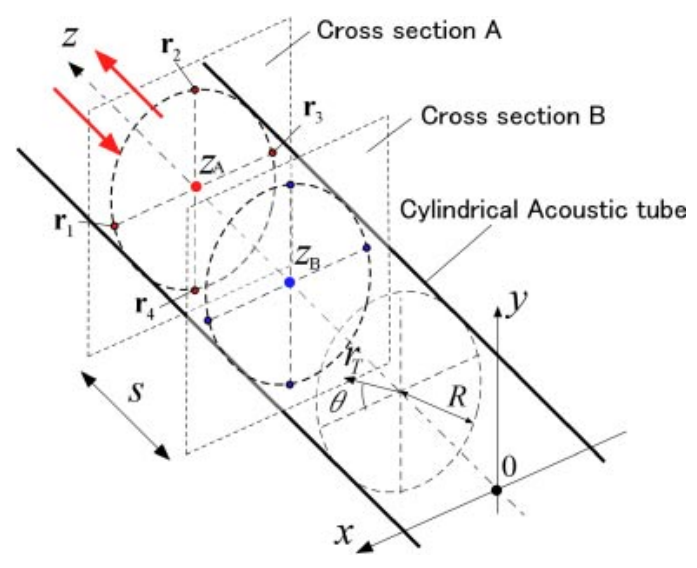

Fig. 1 Sound field in the tube.

$$
\begin{aligned}
& p\left(r_{\mathrm{T}}, \theta, z, t\right) \\
& =\sum_{m=0}^{\infty} \sum_{n=0}^{\infty} C_{m n} J_{m}\left(k_{r(m, n)} r_{\mathrm{T}}\right) \\
& \quad \times\left[e^{-j m \theta}\left(A_{m n}^{\sigma} e^{-j k_{z(m, n) z}}+B_{m n}^{\sigma} e^{j k_{z(m, n)} z}\right)\right. \\
& \left.\quad+e^{j m \theta}\left(A_{m n}^{\tau} e^{-j k_{z(m, n) z}}+B_{m n}^{\tau} e^{j k_{z(m, n) z}}\right)\right] e^{j \omega t},
\end{aligned}
$$

where $r_{\mathrm{T}}$ denotes the distance from the center of the circular cross section and $\theta$ is the angle from the $x$-axis. $J_{m}$ is the Bessel function of the first kind. $m$ and $n$ denote the acoustic modal order in the circumferential direction and in the radial direction, respectively. $A_{m n}^{\sigma}, B^{\sigma}{ }_{m n}, A^{\tau}{ }_{m n}$, and $B_{m n}^{\tau}$ are the amplitudes of the waves. $k_{z(m, n)}$ is the wave number of the $(m, n)$ mode in the $z$-direction: $k_{z(m, n)}=$ $\left(k_{0}^{2}-k_{r(m, n)}\right)^{1 / 2}$, where $k_{0}=\omega / c$ (c: sound speed), and $k_{r(m, n)}$ is the wave number in the cross section of the tube and satisfies the boundary condition at the inner surface of the tube. $k_{r(m, n)} R$ that satisfies the boundary condition is expressed as $\lambda_{m, n}$. Figure 2 shows each acoustic mode and the corresponding value of $\lambda_{m, n}$. $C_{m n}$ is a normalization factor as following equation is satisfied.

$$
C_{m n}^{2} \int_{S} J_{m}^{2}\left(k_{r(m, n)} r\right) d s=S,
$$

where $S$ is area of the cross section of the tube.

In the proposed method, four measurement points, indicated as $\boldsymbol{r}_{1}, \boldsymbol{r}_{2}, \boldsymbol{r}_{3}$, and $\boldsymbol{r}_{4}$ in Fig. 1, are considered. These points are located on the inner surface of the tube at every quarter of the perimeter. They are $\boldsymbol{r}_{1}=(R, 0, z), \boldsymbol{r}_{2}=$ $(R, \pi / 2, z), \boldsymbol{r}_{3}=(R, \pi, z)$, and $\boldsymbol{r}_{4}=(R, 3 \pi / 2, z)$ in cylindrical coordinates. The proposed method considers the frequency range below $f_{c 0,1}$ in which the waves of the $(0,0),(1,0)$, and $(2,0)$ modes propagate.

In order to extract the normal-propagating factor, the sum of the pressure signals at these measurement points is considered as proposed in the authors' previous paper [4,5]. That can be derived from Eq. (1) as follows:

$$
\begin{aligned}
p_{\mathrm{s}} & =p\left(\boldsymbol{r}_{1}\right)+p\left(\boldsymbol{r}_{2}\right)+p\left(\boldsymbol{r}_{3}\right)+p\left(\boldsymbol{r}_{4}\right) \\
& =4 C_{00}\left(A_{00} e^{-j k_{0} z}+B_{00} e^{+j k_{0} z}\right) e^{j \omega t} .
\end{aligned}
$$

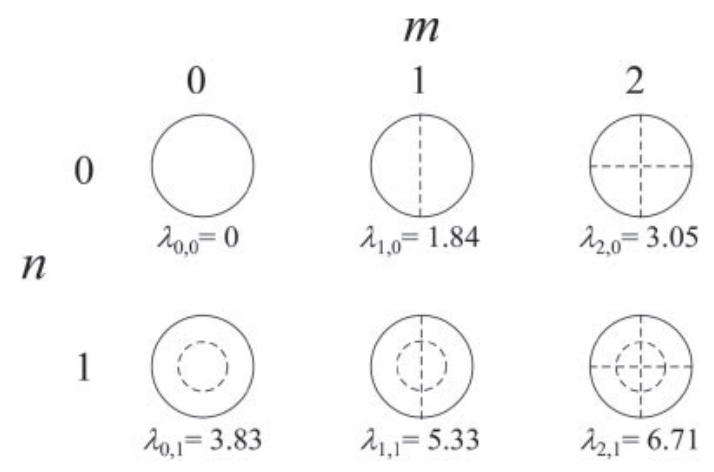

Fig. 2 Acoustic modes. 
This equation implies that summing the signals cancels the signals due to the $(1,0)$ and $(2,0)$ modes and yields the normal-propagating factor. The sums of the measurement signals at the four points are obtained for two cross sections, A and B in Fig. 1, and the normal-propagating factor on each cross section are extracted. Then, a frequency response function for the normal-propagating factors between two cross sections can be calculated as $H_{\mathrm{AB}}=P_{\mathrm{sB}} / P_{\mathrm{sA}}$, where $P_{\mathrm{SA}}$ and $P_{\mathrm{sB}}$ are the frequency spectrums of the sum of the measurement signals at cross sections $\mathrm{A}$ and $\mathrm{B}$, respectively. Using this frequency response function, the normal-incidence sound absorption coefficient can be obtained.

The proposed method is based on the assumption that the amount of scattering on a specimen surface is not large. If a large amount of scattering exists, the normal-incident sound wave may be reflected obliquely in the frequency region in which the higher-order acoustic modes can propagate in the tube. In other words, the $(0,0)$ mode can be converted to the other higher-order modes due to the scattering at the specimen. In this case, it is considered that the normal-incidence absorption coefficient cannot be measured accurately even using the proposed eight-microphone method. Hence, in order to confirm the reliability of measured results, a monitoring method of the scattering effects is investigated in this study.

Here, the sound pressure difference between the signals of the two microphones placed on opposite sides of the tube is considered at the cross section A. $\Delta p_{\mathrm{hA}}$ is the difference between $p\left(\boldsymbol{r}_{1}\right)$ and $p\left(\boldsymbol{r}_{3}\right)$ and $\Delta p_{\mathrm{vA}}$ is the one between $p\left(\boldsymbol{r}_{2}\right)$ and $p\left(\boldsymbol{r}_{4}\right)$. These can be expressed from Eq. (1) as follows:

$$
\begin{aligned}
\Delta p_{\mathrm{hA}}= & p\left(\boldsymbol{r}_{1}\right)-p\left(\boldsymbol{r}_{3}\right) \\
= & 2 e^{j \omega t} C_{10} J_{1}\left(\lambda_{10}\right) \\
& \times\left\{\left(A_{10}^{\sigma}+A_{10}^{\tau}\right) e^{-j k_{z(1,0) z_{\mathrm{A}}}}+\left(B_{10}^{\sigma}+B_{10}^{\tau} e\right) e^{j k_{z(1,0) z_{\mathrm{A}}}}\right\},
\end{aligned}
$$

$$
\begin{aligned}
\Delta p_{\mathrm{vA}}= & p\left(\boldsymbol{r}_{2}\right)-p\left(\boldsymbol{r}_{4}\right) \\
= & -2 j e^{j \omega t} C_{10} J_{1}\left(\lambda_{10}\right) \\
& \times\left\{\left(A_{10}^{\sigma}-A_{10}^{\tau}\right) e^{-j k_{z(1,0) z_{\mathrm{A}}}}+\left(B_{10}^{\sigma}-B_{10}^{\tau}\right) e^{j k_{z(1,0) z_{\mathrm{A}}}}\right\} .
\end{aligned}
$$

These equations imply that taking the difference between the two microphone signals at two opposing points cancels the signals from $(0,0)$ and $(2,0)$ modes and extracts only the signal from $(1,0)$ mode. If the measurement apparatus has a perfect symmetry, the $(1,0)$ modes should be caused by the scattering at the specimen. Hence, this extracted $(1,0)$ mode signal indicates the existence of the scattering at the specimen.

\subsection{Reflection Coefficient}

Next, for the quantitative measurement of the scattering effect, the reflection coefficient in the tube is considered. In the target frequency range of the proposed normalincidence absorption coefficient measurement method, the $(0,0),(1,0)$, and $(2,0)$ modes exist in the tube. Their complex amplitudes are expressed as $A_{00}, B_{00}, A^{\sigma}{ }_{10}, A^{\tau}{ }_{10}$, $B^{\sigma}{ }_{10}, B^{\tau}{ }_{10}, A^{\sigma}{ }_{20}, A^{\tau}{ }_{20}, B^{\sigma}{ }_{20}$, and $B^{\tau}{ }_{20}$, where $A$ and $B$ represent the reflected and incident wave amplitudes, superscript $\sigma$ and $\tau$ denote $\theta+$ and $\theta-$ directions. In this study, the complex ratios of the amplitude of the reflected $(1,0)$ mode to that of the incident $(0,0)$ mode, which is $r^{\sigma}{ }_{0 \rightarrow 1}=A^{\sigma}{ }_{10} / B_{00}$ and $r_{0 \rightarrow 1}^{\tau}=A_{10}^{\tau} / B_{00}$ are considered and they are called normal-incidence $(1,0)$-mode-reflection coefficients for the sake of simplicity. Strictly speaking, $r^{\sigma}{ }_{0 \rightarrow 1}$ and $r^{\tau}{ }_{0 \rightarrow 1}$ are not normal-incidence $(1,0)$-modereflection coefficients because $A^{\sigma}{ }_{10}$ and $A^{\tau}{ }_{10}$ consist of not only the wave reflected from the incident $(0,0)$ mode but also the waves reflected from the incident higher order modes.

On the cross section A, the frequency response function of $\Delta p_{\mathrm{hA}}$ to $p_{\mathrm{sA}}$ and that of $\Delta p_{\mathrm{vA}}$ to $p_{\mathrm{sA}}$ are expressed from Eqs. (3), (4), and (5) as

$$
\begin{aligned}
H_{\mathrm{hA}}= & \frac{\Delta p_{\mathrm{hA}}}{p_{\mathrm{sA}}} \\
= & \frac{C_{10} J_{1}\left(\lambda_{10}\right)}{2 C_{00}} \\
& \times \frac{\left(r_{0 \rightarrow 1}^{\sigma}+r_{0 \rightarrow 1}^{\tau}\right) e^{-j k_{z(1,0) z_{\mathrm{A}}}}+\left(\beta_{0 \rightarrow 1}^{\sigma}+\beta_{0 \rightarrow 1}^{\tau}\right) e^{j k_{z(1,0) z_{\mathrm{A}}}}}{r_{0 \rightarrow 0} e^{-j k_{0} z_{\mathrm{A}}}+e^{j k_{0} z_{\mathrm{A}}}},
\end{aligned}
$$

$$
\begin{aligned}
H_{\mathrm{vA}}= & \frac{\Delta p_{\mathrm{vA}}}{p_{\mathrm{sA}}} \\
= & -\frac{j C_{10} J_{1}\left(\lambda_{10}\right)}{2 C_{00}} \\
& \times \frac{\left(r_{0 \rightarrow 1}^{\sigma}-r_{0 \rightarrow 1}^{\tau}\right) e^{-j k_{z(1,0) z_{\mathrm{A}}}}+\left(\beta_{0 \rightarrow 1}^{\sigma}-\beta_{0 \rightarrow 1}^{\tau}\right) e^{j k_{z(1,0) z_{\mathrm{A}}}}}{r_{0 \rightarrow 0} e^{-j k_{0} z_{\mathrm{A}}}+e^{j k_{0} z_{\mathrm{A}}}},
\end{aligned}
$$

where $r_{0 \rightarrow 0}=A_{00} / B_{00}$ denotes a normal-incidence reflection coefficient, which is obtained from the frequency response function $H_{\mathrm{AB}}$ as explained in the authors' previous study [4,5]. And $\beta^{\sigma}{ }_{0 \rightarrow 1}=B^{\sigma}{ }_{10} / B_{00}$ and $\beta^{\tau}{ }_{0 \rightarrow 1}=$ $B^{\tau}{ }_{10} / B_{00}$ denote ratios of the incidence $(1,0)$ mode to the incident $(0,0)$ mode in the $\sigma$ direction and that in the $\tau$ direction, respectively.

In the same way, $H_{\mathrm{hB}}$ and $H_{\mathrm{vB}}$ can be derived on the cross section $\mathrm{B}$. Then, the pressure reflection coefficient of the reflected $(1,0)$ mode to the incident $(0,0)$ mode can be derived as follows:

$$
\begin{aligned}
& r_{0 \rightarrow 1}^{\sigma}=\frac{C_{00}}{C_{10} J_{1}\left(\lambda_{10}\right)} \\
& \times\left[\frac{\left(r_{0 \rightarrow 0} e^{-j k_{0} z_{\mathrm{A}}}+e^{j k_{0} z_{\mathrm{A}}}\right) e^{j k_{z(1,0) z_{\mathrm{B}}}}\left(H_{\mathrm{hA}}+j H_{\mathrm{vA}}\right)}{e^{-j k_{z(1,0)}\left(z_{\mathrm{A}}-z_{\mathrm{B}}\right)}-e^{j k_{z(1,0)}\left(z_{\mathrm{A}}-z_{\mathrm{B}}\right)}}\right.
\end{aligned}
$$




$$
\begin{aligned}
& \left.-\frac{\left(r_{0 \rightarrow 0} e^{-j k_{0} z_{\mathrm{B}}}+e^{j k_{0} z_{\mathrm{B}}}\right) e^{j k_{z(1,0)} z_{\mathrm{A}}}\left(H_{\mathrm{hB}}+j H_{\mathrm{vB}}\right)}{e^{-j k_{z(1,0)}\left(z_{\mathrm{A}}-z_{\mathrm{B}}\right)}-e^{j k_{z(1,0)}\left(z_{\mathrm{A}}-z_{\mathrm{B}}\right)}}\right], \\
r_{0 \rightarrow 1}^{\tau} & =\frac{C_{00}}{C_{10} J_{1}\left(\lambda_{10}\right)} \\
\times & {\left[\frac{\left(r_{0 \rightarrow 0} e^{-j k_{0} z_{\mathrm{A}}}+e^{j k_{0} z_{\mathrm{A}}}\right) e^{j k_{z(1,0)} z_{\mathrm{B}}}\left(H_{\mathrm{hA}}-j H_{\mathrm{vA}}\right)}{e^{-j k_{z(1,0)}\left(z_{\mathrm{A}}-z_{\mathrm{B}}\right)}-e^{j k_{z(1,0)}\left(z_{\mathrm{A}}-z_{\mathrm{B}}\right)}}\right.} \\
& \left.-\frac{\left(r_{0 \rightarrow 0} e^{-j k_{0} z_{\mathrm{B}}}+e^{j k_{0} z_{\mathrm{B}}}\right) e^{j k_{z(1,0)} z_{\mathrm{A}}}\left(H_{\mathrm{hB}}-j H_{\mathrm{vB}}\right)}{e^{-j k_{z(1,0)}\left(z_{\mathrm{A}}-z_{\mathrm{B}}\right)}-e^{j k_{z(1,0)}\left(z_{\mathrm{A}}-z_{\mathrm{B}}\right)}}\right] .
\end{aligned}
$$

Similarly, the ratios of the incident $(1,0)$ mode to the incident $(0,0)$ mode are derived as

$$
\begin{aligned}
\beta_{0 \rightarrow 1}^{\sigma} & =\frac{C_{00}}{C_{10} J_{1}\left(\lambda_{10}\right)} \\
\times & {\left[\frac{\left(r_{0 \rightarrow 0} e^{-j k_{0} z_{\mathrm{A}}}+e^{j k_{0} z_{\mathrm{A}}}\right) e^{-j k_{z(1,0)} z_{\mathrm{B}}}\left(H_{\mathrm{hA}}+j H_{\mathrm{vA}}\right)}{e^{j k_{z(1,0)}\left(z_{\mathrm{A}}-z_{\mathrm{B}}\right)}-e^{-j k_{z(1,0)}\left(z_{\mathrm{A}}-z_{\mathrm{B}}\right)}}\right.} \\
& \left.-\frac{\left(r_{0 \rightarrow 0} e^{-j k_{0} z_{\mathrm{B}}}+e^{j k_{0} z_{\mathrm{B}}}\right) e^{-j k_{z(1,0)} z_{\mathrm{A}}}\left(H_{\mathrm{hB}}+j H_{\mathrm{vB}}\right)}{e^{j k_{z(1,0)}\left(z_{\mathrm{A}}-z_{\mathrm{B}}\right)}-e^{-j k_{z(1,0)}\left(z_{\mathrm{A}}-z_{\mathrm{B}}\right)}}\right],
\end{aligned}
$$

$$
\begin{aligned}
\beta_{0 \rightarrow 1}^{\tau} & =\frac{C_{00}}{C_{10} J_{1}\left(\lambda_{10}\right)} \\
\times & {\left[\frac{\left(r_{0 \rightarrow 0} e^{-j k_{0} z_{\mathrm{A}}}+e^{j k_{0} z_{\mathrm{A}}}\right) e^{-j k_{z(1,0)} z_{\mathrm{B}}}\left(H_{\mathrm{hA}}-j H_{\mathrm{vA}}\right)}{e^{j k_{z(1,0)}\left(z_{\mathrm{A}}-z_{\mathrm{B}}\right)}-e^{-j k_{z(1,0)}\left(z_{\mathrm{A}}-z_{\mathrm{B}}\right)}}\right.} \\
& \left.-\frac{\left(r_{0 \rightarrow 0} e^{-j k_{0} z_{\mathrm{B}}}+e^{j k_{0} z_{\mathrm{B}}}\right) e^{-j k_{z(1,0)} z_{\mathrm{A}}}\left(H_{\mathrm{hB}}-j H_{\mathrm{vB}}\right)}{e^{j k_{z(1,0)}\left(z_{\mathrm{A}}-z_{\mathrm{B}}\right)}-e^{-j k_{z(1,0)}\left(z_{\mathrm{A}}-z_{\mathrm{B}}\right)}}\right] .
\end{aligned}
$$

The sound pressure and the particle velocity of the incident sound wave in this case are as follows:

$$
\begin{aligned}
p_{\mathrm{i}}= & e^{j \omega t}\left[C_{00} B_{00} e^{j k_{0} z}+C_{10} J_{1}\left(\frac{\lambda_{10}}{R} r\right) e^{-j \theta} B_{10}^{\sigma} e^{j k_{z(1,0)} z}\right. \\
& \left.+C_{10} J_{1}\left(\frac{\lambda_{10}}{R} r\right) e^{j \theta} B_{10}^{\tau} e^{j k_{z(1,0) z}}\right], \\
v_{\mathrm{i}}= & \frac{e^{j \omega t}}{\rho c}\left[C_{00} B_{00} e^{j k_{0} z}+C_{10} J_{1}\left(\frac{\lambda_{10}}{R} r\right) e^{-j \theta} B_{10}^{\sigma} e^{j k_{z(1,0)} z} \frac{k_{z(1,0)}}{k_{0}}\right. \\
+ & \left.C_{10} J_{1}\left(\frac{\lambda_{10}}{R} r\right) e^{j \theta} B_{10}^{\tau} e^{j k_{z(1,0)} z} \frac{k_{z(1,0)}}{k_{0}}\right] .
\end{aligned}
$$

Hence, the incident sound power to a specimen is derived as follows:

$$
\begin{aligned}
W_{\mathrm{i}} & =\frac{1}{2} \int_{S} \operatorname{Re}\left(p_{\mathrm{i}} v_{\mathrm{i}}^{*}\right) d s \\
& =\frac{S}{2 \rho c}\left[\left|B_{00}\right|^{2}+\frac{k_{z(1,0)}}{k_{0}}\left(\left|B_{10}^{\sigma}\right|^{2}+\left|B_{10}^{\tau}\right|^{2}\right)\right] .
\end{aligned}
$$

Similarly, the reflected sound power is obtained as follows:

$$
W_{\mathrm{r}}=\frac{1}{2} \int_{S} \operatorname{Re}\left(p_{\mathrm{r}} v_{\mathrm{r}}^{*}\right) d s
$$

$$
=\frac{S}{2 \rho c}\left[\left|A_{00}\right|^{2}+\frac{k_{z(1,0)}}{k_{0}}\left(\left|A_{10}^{\sigma}\right|^{2}+\left|A_{10}^{\tau}\right|^{2}\right)\right],
$$

where $p_{\mathrm{r}}$ and $v_{\mathrm{r}}$ are the sound pressure and the particle velocity of the reflected sound wave, respectively.

From Eqs. (14) and (15), a total power reflection coefficient in a tube can be calculated as follows:

$$
R_{\mathrm{total}}=\frac{R_{0 \rightarrow 0}+R_{0 \rightarrow 1}}{1+B_{0 \rightarrow 1}},
$$

where,

$$
\begin{aligned}
& R_{0 \rightarrow 0}=\left|r_{0 \rightarrow 0}\right|^{2} \\
& R_{0 \rightarrow 1}=\frac{k_{z(1,0)}}{k_{0}}\left(\left|r_{0 \rightarrow 1}^{\sigma}\right|^{2}+\left|r_{0 \rightarrow 1}^{\tau}\right|^{2}\right),
\end{aligned}
$$

and

$$
B_{0 \rightarrow 1}=\frac{k_{z(1,0)}}{k_{0}}\left(\left|\beta_{0 \rightarrow 1}^{\sigma}\right|^{2}+\left|\beta_{0 \rightarrow 1}^{\tau}\right|^{2}\right) .
$$

$R_{0 \rightarrow 0}$ denotes a normal-incidence power reflection coefficient, which can be measured with the proposed eightmicrophone method. $R_{0 \rightarrow 1}$ denotes a power reflection coefficients of the $(1,0)$ mode to the incident $(0,0)$ mode, which is called a normal-incidence $(1,0)$-mode power reflection coefficient in this study. $B_{0 \rightarrow 1}$ denotes a power ratio of the incident $(1,0)$ mode to the incident $(0,0)$ mode.

At last, by measuring the frequency response functions, $H_{\mathrm{hA}}, H_{\mathrm{vA}}, H_{\mathrm{hB}}$, and $H_{\mathrm{vB}}$, the normal-incidence $(1,0)$-mode power reflection coefficient $R_{0 \rightarrow 1}$ and the total power reflection coefficient in the tube $R_{\text {total }}$ can be calculated.

When the scattering does not occur, $R_{0 \rightarrow 1}$ is zero and $R_{0 \rightarrow 0}$ is identical to $R_{\text {total }}$. On the other hand, when the amount of the scattering increases, $R_{0 \rightarrow 1}$ has some value, and $R_{0 \rightarrow 0}$ and $R_{\text {total }}$ take different values. That difference indicates the amount of the scattering effect in the measured value of the normal-incidence absorption coefficient.

Hence, if you monitor $R_{0 \rightarrow 1}$ or $R_{\text {total }}$ during measurement of normal-incidence sound absorption coefficient, the measurement reliability can be quantitatively evaluated. In other words, by monitoring $R_{0 \rightarrow 1}$ or $R_{\text {total }}$, it is possible to judge whether a measured result is reliable or not.

\section{NUMERICAL SIMULATION}

\subsection{Numerical Simulation Model}

In order to confirm whether the proposed method can work when performing the actual absorption coefficient measurements, the numerical simulations were conducted. Figures 3 and 4 show the calculation conditions and the finite element (FE) model, respectively. As an extreme case, the case in which the test specimen is rigid plate is considered to investigate the effect of the scattering. This is the case that the incident wave is not absorbed and totally reflected at the specimen. 


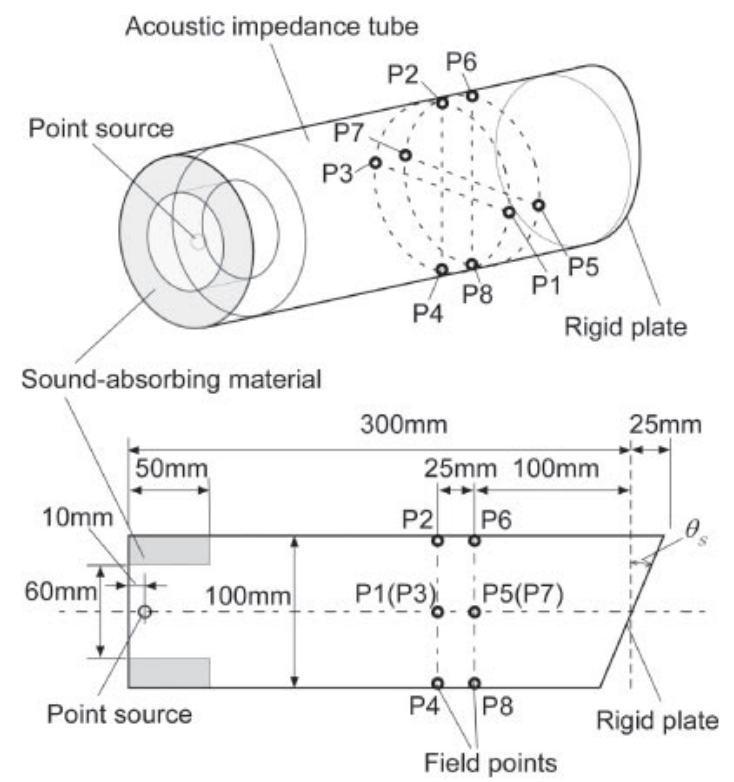

Fig. 3 Calculation conditions.

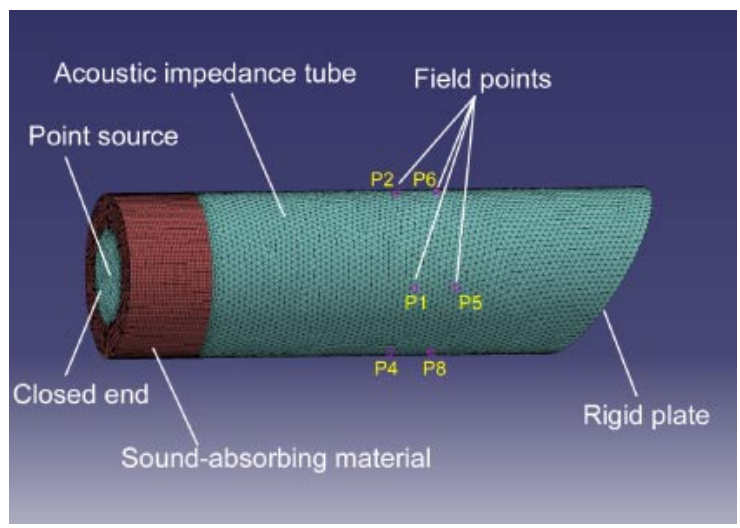

Fig. 4 FE model.

The calculation model is based on the experimental conditions stated later. The FEM is used with LMS Virtual.Lab Acoustics in the calculation. To simulate the actual acoustic field in the impedance tube accurately, a direct approach in the acoustic response analysis, which is based on the physical coordinates, were used. The diameter of the tube is $100 \mathrm{~mm}$. Tetrahedral linear element (TETRA4) was used for the 3D mesh. The number of elements is 247,669 . The maximum calculation frequency, which is determined by the relation between the mesh size and the $1 / 6$ wavelength, is $4,382 \mathrm{~Hz}$. A sound-absorbing material is placed at the source end of the tube to absorb sound waves reflected from the specimen. A point source is placed at a distance of $10 \mathrm{~mm}$ from the end. Eight field points, which correspond to the microphones, are set on the inner surface of the tube in accordance with the theory. These points are indicated as P1, P2, .., and P8 in Fig. 3.

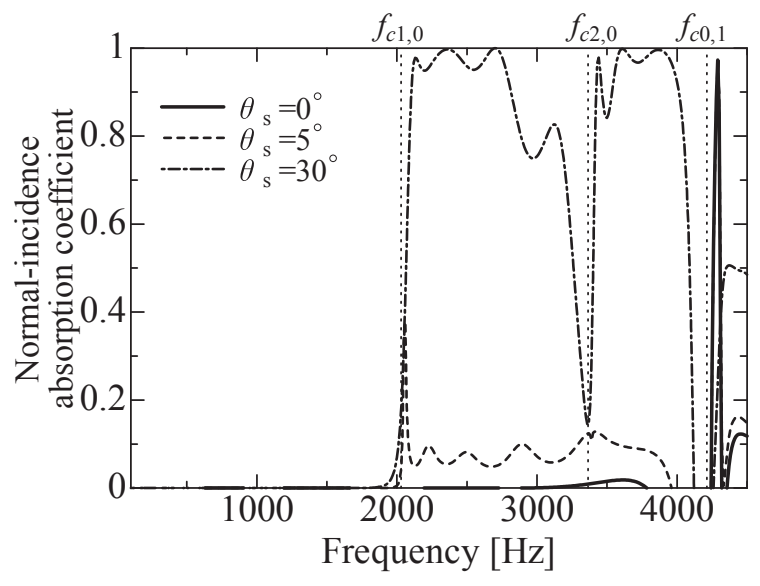

Fig. 5 Calculated normal-incidence absorption coefficient of rigid plates when the inclination angle are 0,5 , and 30 degrees.

The distances of the cross sections, where the field points are located, from the specimen surface are 100 and $125 \mathrm{~mm}$. The frequency response functions, $H_{\mathrm{AB}}, H_{\mathrm{hA}}$, $H_{\mathrm{vA}}, H_{\mathrm{hB}}$, and $H_{\mathrm{vB}}$, were calculated from the sound pressures at these field points, obtaining the absorption coefficients and the reflection coefficients using Eqs. (16) to (19).

The rigid plate as a specimen is modeled by a rigid boundary condition. To generate the scattering, the surface of the plate is inclined at some angles to the cross section of the tube. The inclination angle of the rigid plate to the cross section is $\theta_{\mathrm{s}}$. The simulations were conducted in the cases of $\theta_{\mathrm{s}}=0,5$, and $30^{\circ}$.

\subsection{Simulation Results}

Figures 5 and 6 show the calculated normal-incidence absorption coefficient and the power reflection coefficient of the rigid plates, which is inclined by 0,5 , and $30^{\circ}$, respectively. When the specimen is not inclined $\left(\theta_{\mathrm{s}}=0^{\circ}\right)$, the numerically calculated absorption coefficient and $R_{0 \rightarrow 1}$ are close to zero. In this case, normal-incidence absorption coefficient and $R_{0 \rightarrow 1}$ are theoretically zero. Hence, these calculated results may be reasonable and imply the effectiveness of the calculation model.

It can be confirmed that in all cases, below the cut-on frequency of the $(1,0)$ mode, the normal-incidence absorption coefficients are zero and $R_{0 \rightarrow 1}$ are also zero. This is because only the $(0,0)$ mode can exist in the tube in that frequency range. In contrast, above the cut-on frequency of the $(1,0)$ mode, in the cases of $\theta_{\mathrm{s}}=5$ and $30^{\circ}$, some absorption coefficients are calculated, although the actual absorption of the rigid plate is of course zero. Besides, as the inclination angle increases, the calculated absorption coefficient increases. 


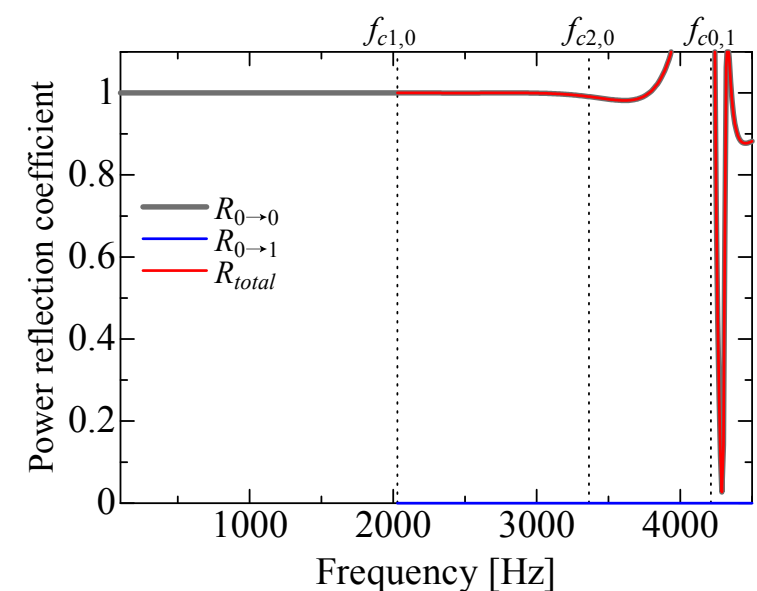

(a) $\theta_{s}=0^{\circ}$

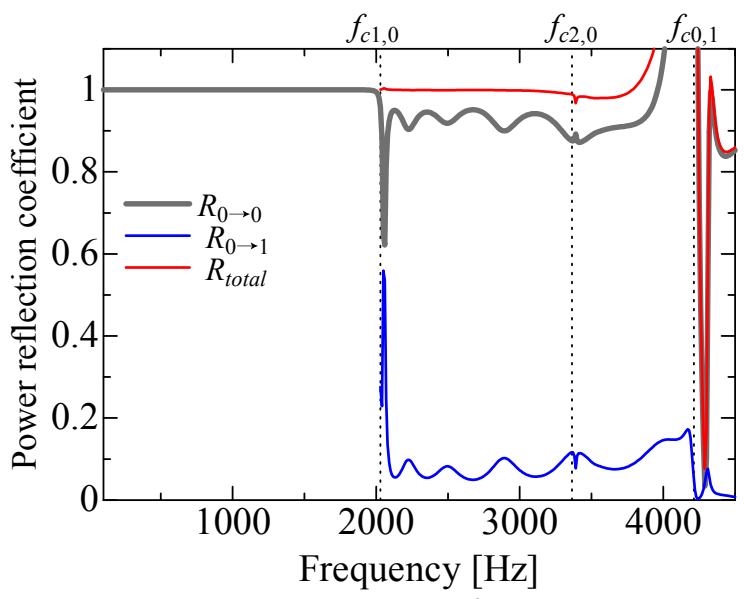

(b) $\theta_{s}=5^{\circ}$

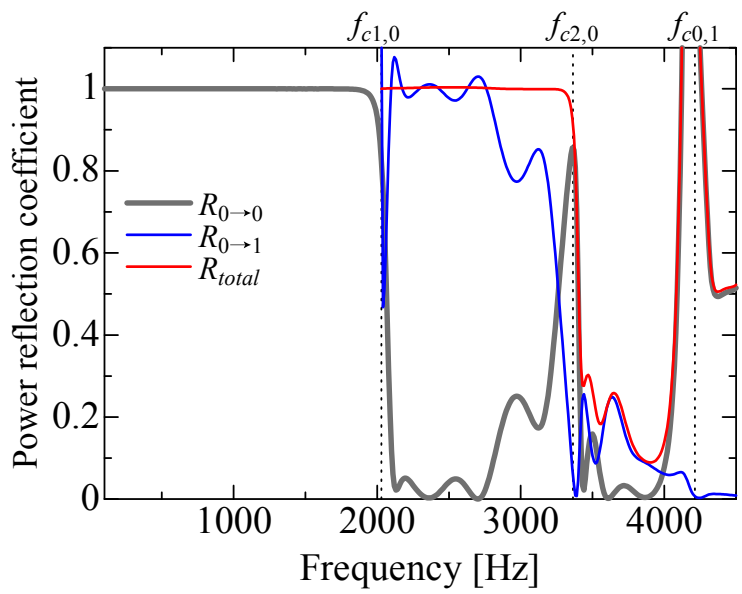

(c) $\theta_{s}=30^{\circ}$

Fig. 6 Calculated power reflection coefficient of the rigid plates.

In the cases that the inclination angles are 5 and $30^{\circ}$, some amount of $R_{0 \rightarrow 1}$ is observed. This implies that the scattering occurs. Especially, in the case of $30^{\circ}$, the high $R_{0 \rightarrow 1}$ is observed in the frequency range in which a high normal-incidence absorption coefficient is observed. These results imply that in the case when the inclination angle is large, some amount of normal-incident sound wave is

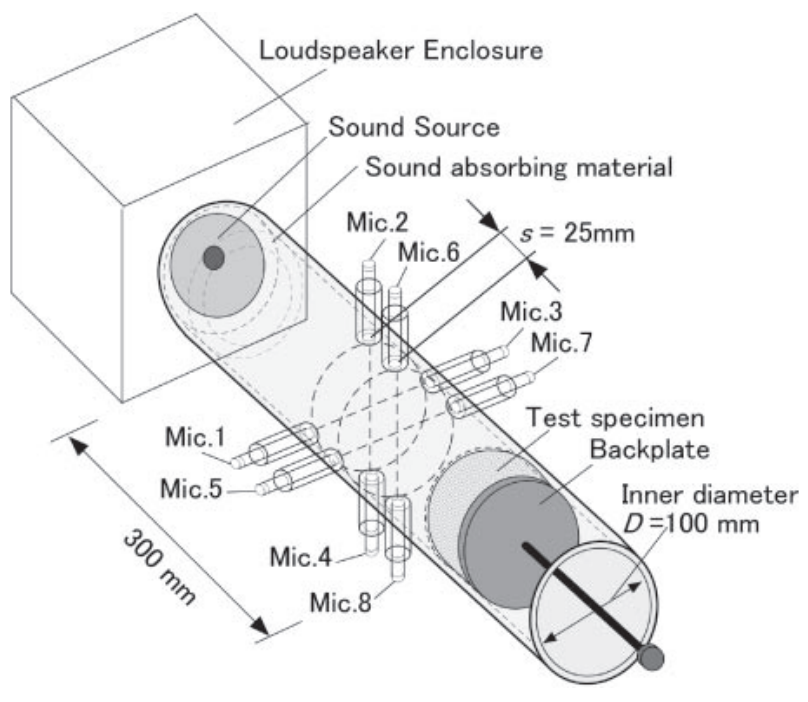

Fig. 7 Experimental set-up.

reflected at the rigid plate obliquely and the obliquely reflected wave cannot be sensed with the proposed method because the proposed eight microphone method only sense the normal-propagating factor. Hence, the apparent absorption coefficient is calculated.

In addition, the total power reflection coefficient is close to one below the $(2,0)$ mode cut-on frequency in all cases. These results are also physically reasonable and prove the reliability of the proposed monitoring method.

\section{EXPERIMENT}

\subsection{Experimental Setup}

In order to verify the validity and the feasibility of the proposed monitoring method, experiments were conducted. Figure 7 shows a schematic view of the experimental setup. The acoustic impedance tube is made from acrylic and the inner diameter $D$ is $100 \mathrm{~mm}$. The upper frequency limit which is determined by the microphone distance $(s=25 \mathrm{~mm})$ is $6,237 \mathrm{~Hz}$ (in the case of $c=346.5 \mathrm{~m} / \mathrm{s}$ ). Eight 1/4-inch microphones were used and were positioned in accordance with the theory. White noise was used as the noise source.

\subsection{Experimental Results}

First, the case in which the incident sound wave is totally reflected is considered. Although in the simulation the rigid plate was targeted, oil clay was chosen as a test specimen in the experiment because the oil clay might not absorb sound and is easy to install with some inclination angle in the tube. Hence, this is the case corresponding to the simulations on the rigid plates showed in the former chapter. Figure 8 shows the photograph of the oil clay which was inserted in the sample holder. The surface of the oil clay was inclined by 30 degrees. 


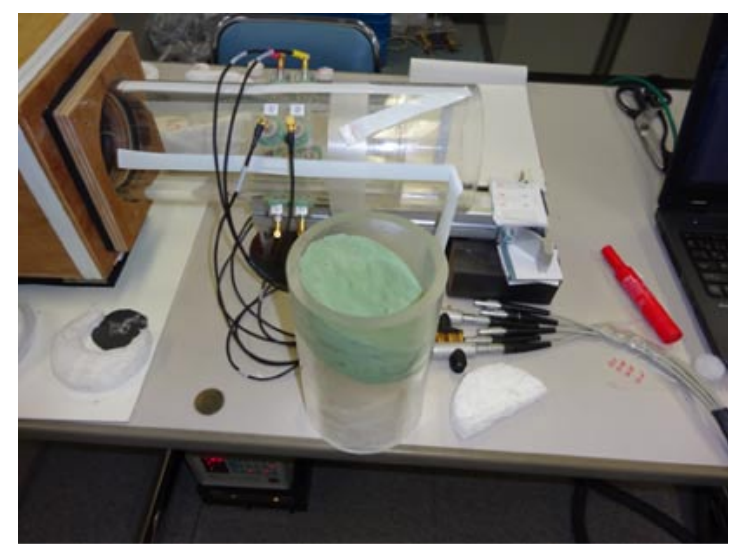

Fig. 8 Photograph of the oil clay which was inserted in the sample holder.

Figure 9(a) shows the measured normal-incidence absorption coefficients using the proposed eight-microphone method in the cases that the inclination angles are 0 and $30^{\circ}$. When the surface is not inclined, the measured absorption coefficient is close to zero below about $4,000 \mathrm{~Hz}$. In contrast, when the surface is inclined by $30^{\circ}$, the high absorption coefficient is observed above the cut-on frequency of the $(1,0)$ mode, although below the frequency, the absorption coefficient is nearly zero. Figures 9(b) and 9(c) show the measured power reflection coefficients in these cases, which were measured simultaneously with the normal-incidence absorption measurement. In the case when $\theta_{\mathrm{s}}=0^{\circ}$, the $R_{0 \rightarrow 1}$ is nearly zero, which shows the scattering does not occur. In the case when the surface is inclined by $30^{\circ}$, above the cut-on frequency of the $(1,0)$ mode, high $R_{0 \rightarrow 1}$ is observed and $R_{\text {total }}$ is close to one. This implies that a large amount of the scattering occurs and the incident sound is totally reflected at the specimen. Therefore, it can be confirmed that the apparent absorption coefficient is calculated.

These experimental results are almost coincident with the simulation results. This may prove the theory of monitoring method of the scattering effect, and the validity of the simulation model as well.

Around the cut-on frequency of the $(1,0)$ mode, $R_{0 \rightarrow 1}$ has a value exceeding one in both measured and calculation results. This is the matter on the term definition of the normal-incidence $(1,0)$-mode-reflection coefficient. By the definition, $R_{0 \rightarrow 1}$ includes not only the scattering factor but also the factor of the specular reflection of the incident $(1,0)$ mode. Hence, $R_{0 \rightarrow 1}$ indicates the existence of the scattering, but the value of $R_{0 \rightarrow 1}$ does not directly indicate only the amount of scattering. When the scattering occurs, the normal-incident sound wave is reflected obliquely at the specimen and then the reflected wave comes back to the specimen as the incident $(1,0)$ mode from the source end.

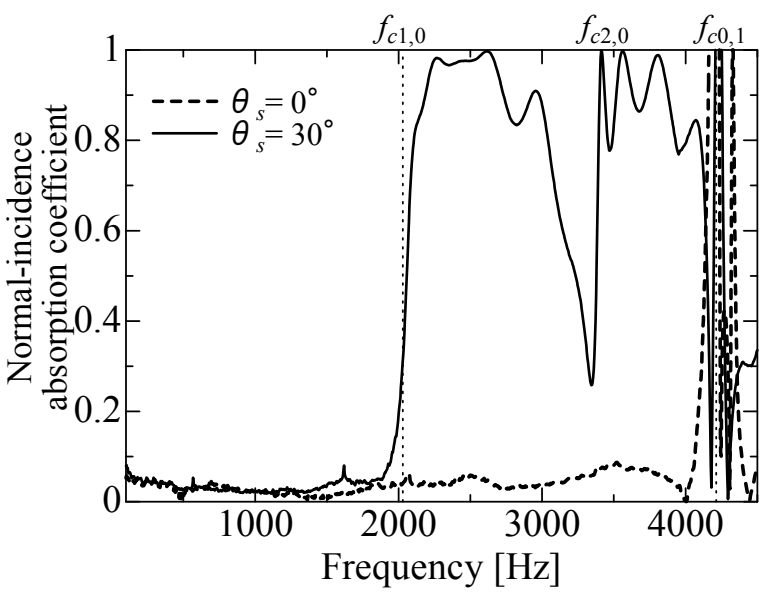

(a) Normal-incidence absorption coefficient

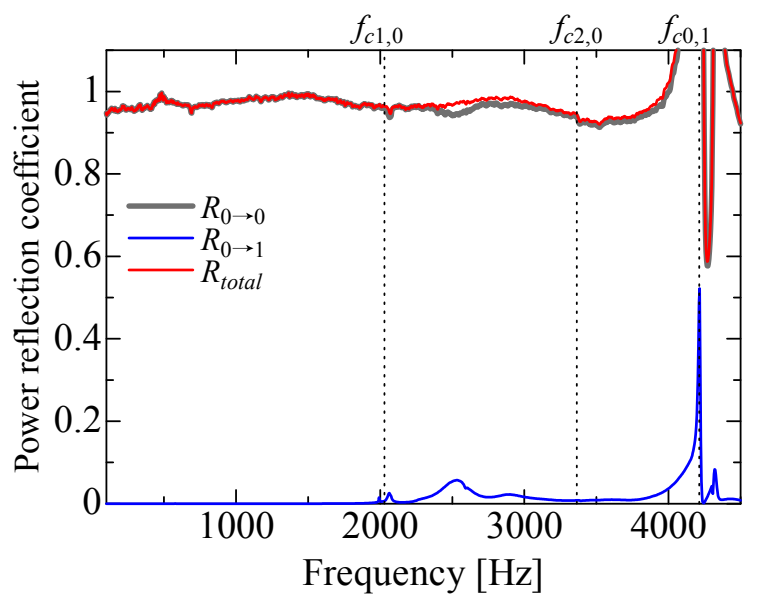

(b) Power reflection coefficient $\left(\theta_{s}=0^{\circ}\right)$

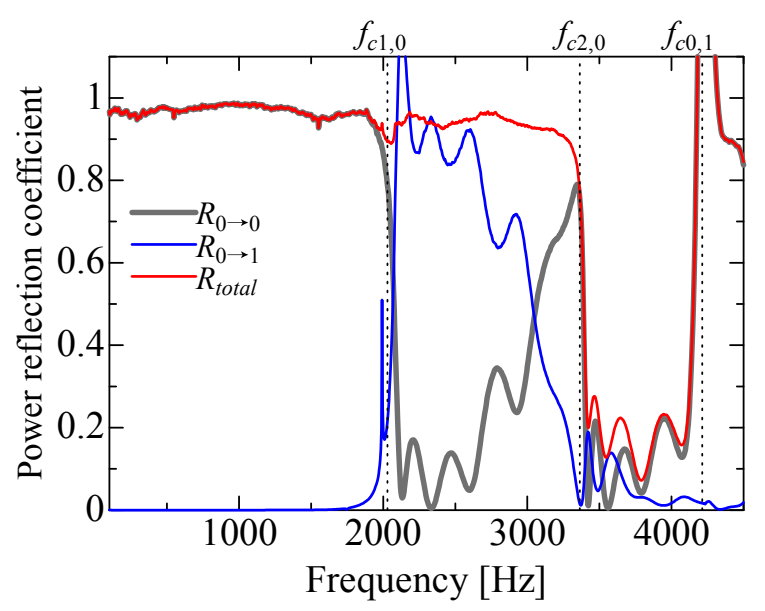

(c) Power reflection coefficient $\left(\theta_{s}=30^{\circ}\right)$

Fig. 9 Measured results in the case of the oil clay.

Since the proposed 8-microphone method cannot sense the $(1,0)$ mode, $R_{0 \rightarrow 0}$ should change. On the other hand, $R_{\text {total }}$ is the ratio of all the reflected power to all the incident power in the tube. It contains all the influence of sound waves in the tube. Therefore, it can be considered that the difference between $R_{\text {total }}$ and $R_{0 \rightarrow 0}$ represents the amount 


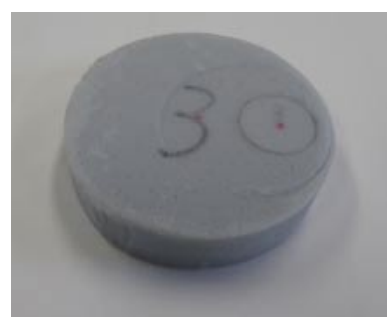

(a) without patch

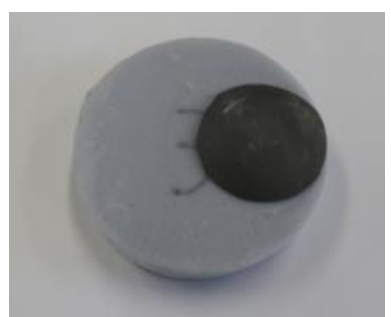

(b) with patch
Fig. 10 Photographs of 25-mm-thick melamine foam on which surface a polypropylene circular patch $(\phi=50$ $\mathrm{mm}, t=0.4 \mathrm{~mm})$ is attached.

of the influence due to the scattering in the absorption coefficient. Because of this, for the purpose of evaluating the amount of the scattering effect quantitatively, it is better to see the difference between $R_{0 \rightarrow 0}$ and $R_{\text {total }}$.

Figure 10 shows the next target specimen: $25-\mathrm{mm}-$ thick melamine foam. Figure 10(b) is in the case when a polypropylene circular patch $(\phi=50 \mathrm{~mm}, t=0.4 \mathrm{~mm})$ was attached at a position shifted from the center. The patch may disturb the acoustic field in the tube, scattering the normal-incident wave in the frequency region in which the higher-order modes can propagate. Figure 10(a) is the normal case which is without the patch.

Figure 11 shows the measured absorption coefficient and the power reflection coefficients of the 25-mm-thick melamine foam in the case which is shown in Fig. 10(a). As Fig. 11(b) shows, $R_{0 \rightarrow 1}$ is small and $R_{0 \rightarrow 0}$ is almost identical to $R_{\text {total }}$ through the frequency range below $f_{c 0,1}$. This implies that the effect of the scattering is very small in measured normal-incidence absorption coefficient. It is considered that in this condition, the scattering at the specimen does not occur. Figure 12 shows the experimental results in the case when the patch is attached as shown in Fig. 10(b). The absorption curve slightly fluctuates above $f_{c 1,0}$, unlike that in Fig. 11. As Fig. 12(b) shows, the value of $R_{0 \rightarrow 1}$ is larger than that in Fig. 11(b) through the frequency range. Moreover, $R_{\text {total }}$ is larger up to 0.053 than $R_{0 \rightarrow 0}$. This difference indicates the amount of the effect of the scattering. This result shows that the effect of the scattering exists but it is small and the measured absorption coefficient is reliable within this degree of difference. It is confirmed that during the absorption measurement, the reliability of measured data can be checked by monitoring $R_{0 \rightarrow 1}$ and $R_{\text {total }}$.

\section{CONCLUSIONS}

The eight-microphone method, which was proposed by the authors, enables the normal-incidence absorption coefficient measurement at about twice the frequency of the conventional two-microphone transfer-function meth-

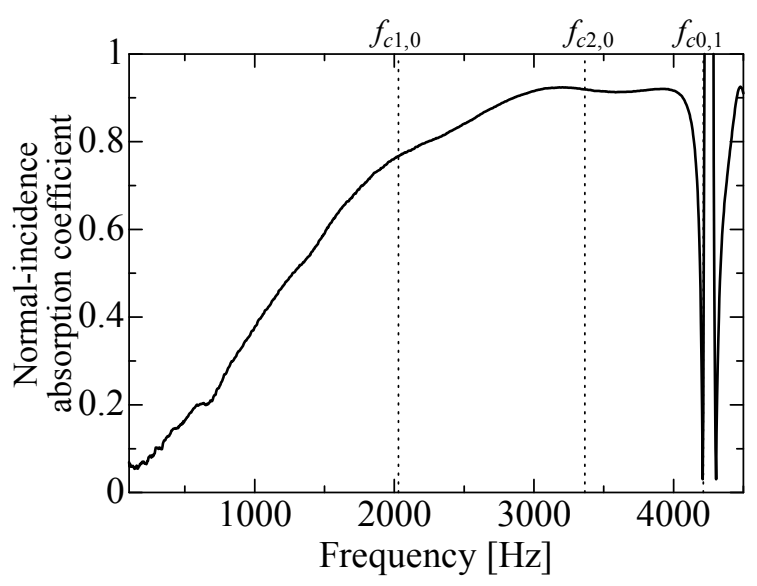

(a) Normal-incidence absorption coefficient

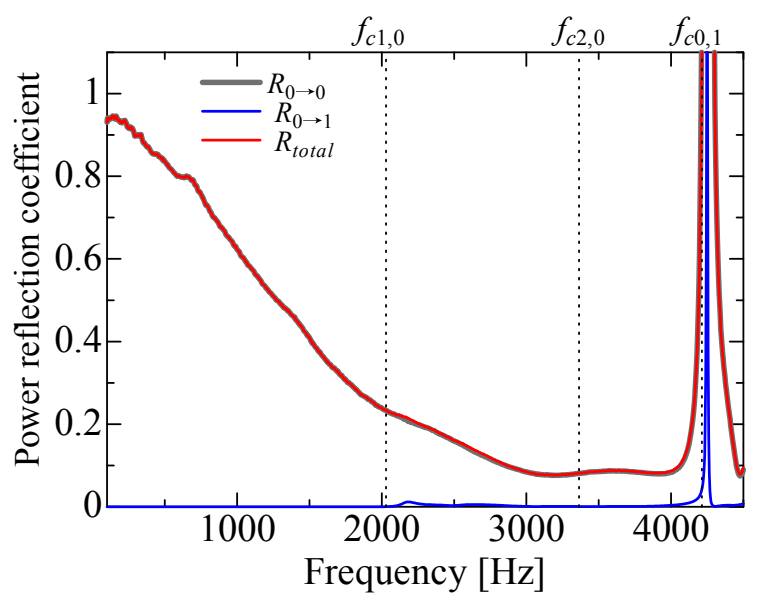

(b) Power reflection coefficient

Fig. 11 Measured results of the 25-mm-thick melamine foam.

od, whereas the method has the limitation which it can work only in the condition that the amount of scattering at the specimen is sufficiently small. If the scattering is large, the normal-incidence absorption coefficient cannot be measured accurately even using the proposed method. Therefore, in practical measurement, the problem of the proposed eight-microphone method is that it is hard to tell how reliable the measured result is. Hence, the monitoring method of the scattering effects on a specimen surface was proposed in this study. The normal-incidence $(1,0)$-mode-reflection coefficient and the total power reflection coefficient in a tube were derived by considering the difference between the signals of the two microphones placed on opposite sides of the tube. The simulations and the experiments were conducted to demonstrate the validity and the feasibility of the proposed method. The theoretical consideration and the experimental results showed that by monitoring the scattering, measurement can be performed as confirming the reliability of the measured result. 


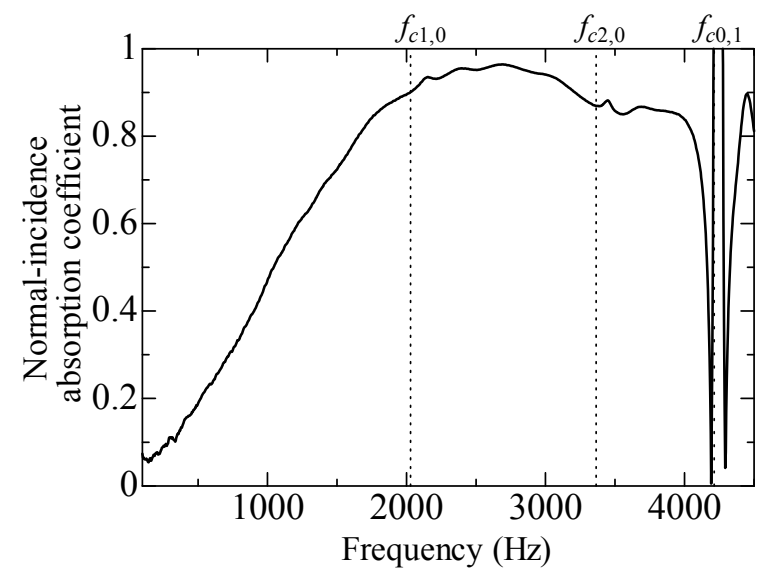

(a) Normal-incidence absorption coefficient

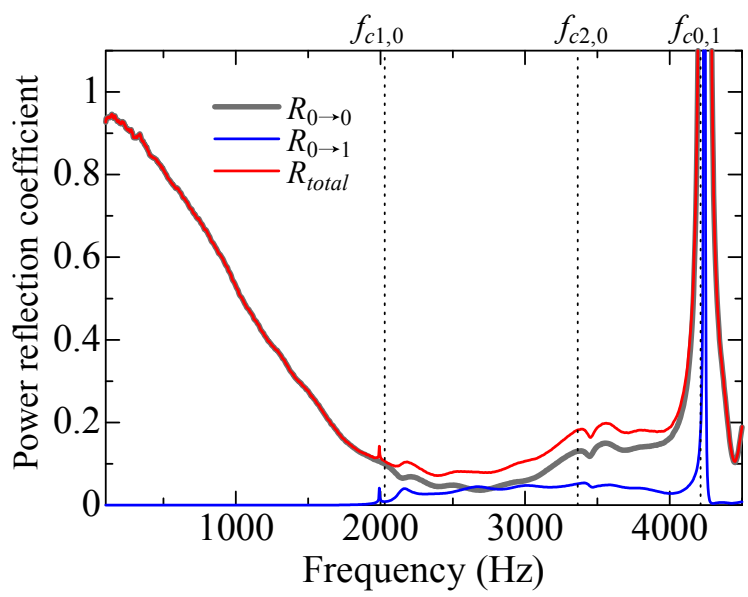

(b) Power reflection coefficient

Fig. 12 Measured results of the 25-mm-thick melamine foam on which surface a polypropylene circular patch is attached at a position shifted from the center.

\section{REFERENCES}

[1] ISO 10534-2, Acoustics - Determination of sound absorption coefficient and impedance in impedance tubes - Part 2: Transfer-function method (1998).

[2] M. Åbom, "Modal decomposition in ducts based on transfer function measurements between microphone pairs," J. Sound Vib., 135, 95-114 (1989).

[3] T. Schultz, L. N. Cattafesta III and M. Sheplak, "Modal decomposition method for acoustic impedance testing in square ducts," J. Acoust. Soc. Am., 120, 3750-3758 (2006).

[4] A. Sanada, "Extension of the frequency range of normal incidence sound absorption coefficient measurement using four or eight microphones," Acoust. Sci. \& Tech., 38, 261-263 (2017).

[5] A. Sanada, K. Iwata and H. Nakagawa, "Extension of the frequency range of normal-incidence sound absorption coefficient measurement in impedance tube using four or eight microphones," Acoust. Sci. \& Tech., 39, 335-342 (2018). 